\title{
Resolviendo mitos sobre indicaciones al paciente durante el blanqueamiento dental
}

\author{
Solving myths about bleaching indications \\ Eric Dario Acuña Navarro ${ }^{1 a}$, Katherine Vilchez Fuentes Rivera ${ }^{1 a}$, Leyla Delgado-Cotrina ${ }^{1 b}$, \\ Lidia Yileng Tay Chu Jon ${ }^{1 c}$
}

\section{RESUMEN}

El blanqueamiento dental es un tratamiento común en pacientes con alta exigencia estética. Existen diversas indicaciones que los profesionales les brindan a sus pacientes para no alterar los resultados y llegar al color deseado por el paciente. Entre ellas tenemos la disminución del consumo de bebidas oscuras (como el té o el café) y el dejar de fumar. Sin embargo, muchas veces estas recomendaciones no tienen sustento en evidencia científica. Encontramos en la literatura estudios que demuestran que estas indicaciones no son totalmente válidas. Es por eso que el objetivo de este artículo de revisión es resaltar la evidencia científica sobre consumo de estos productos durante el blanqueamiento, si es que afectan la efectividad de este tratamiento y aclarar los puntos en los que el clínico general puede tener dudas. Para ello se discutirá la relación del blanqueamiento dental con la ingesta de bebidas, alimentos y hábitos que pueden pigmentar los dientes.

\section{PALABRAS CLAVE: Blanqueamiento de dientes, café, té, vino, hábito de fumar.}

\section{SUMMARY}

Dental bleaching is a common treatment in patients with high aesthetics request. Different indications are given by professionals for improving bleaching results as wished by the patient. Among the directions given to the patients, it's indicated to decrease the consume of dark beverages (like tea or coffee) and avoiding smoke. It's important to note that many of theses indications don't have scientific evidence. Studies show that these indications are not totally real. The purpose of this article is to show the scientific evidence about the consume of these products during bleaching, and if this impact the effectiveness of the treatment and clear up the doubts that the

\footnotetext{
${ }^{1}$ Area de Odontología Restauradora y Estética Facultad de Estomatologia, Universidad Peruana Cayetano Heredia. Lima, Perú.

a Especialidad de Odontología Restauradora y Estética.

b Magister en Odontología

a Doctor en Odontología.
} 
professional may have. The relation between dental bleaching and the consume of drinks, food and habits that may cause pigmentation of teeth.

KEY WORDS: Tooth bleaching, coffee, tea, wine, smoking.

\section{INTRODUCCIÓN}

Es común encontrar pacientes que presenten una elevada exigencia estética en la consulta odontológica. Muchos de ellos solicitan blanqueamiento dental por insatisfacción con el color de sus dientes.

Existen diferentes técnicas para realizar el blanqueamiento dental, todas se basan en el uso de peróxidos de hidrógeno y carbamida en diferentes concentraciones. Una de ellas es la técnica ambulatoria o domiciliara, donde se utilizan productos en bajas concentraciones. Otra técnica es el blanqueamiento en consultorio, donde éstos productos son utilizados en altas concentraciones. Todo procedimiento debe estar siempre supervisado por el odontólogo, quien deberá proporcionar el material y controlar su administración, además de observar el estado de los tejidos blandos del paciente y los cambios sucedidos en la pigmentación o el deterioro de restauraciones preexistentes.

Existen varias recomendaciones que se indican al paciente cuando se realiza este tipo de tratamiento, el profesional suele decir: "No debes tomar café, ni té, ni gaseosas", "No debes fumar" o "el diente se debilita”. Sin embargo, muchas de estas indicaciones no están sustentadas con evidencia científica.

Está demostrado que ciertas sustancias, principalmente alimentos y bebidas oscuras pigmentan los dientes (1), principalmente relacionado a las alteraciones en la superficie del esmalte (2). Sin embargo, diversos autores refieren que el cambio de color de los dientes va a depender de diferentes factores, unos propios a la solución pigmentante y otros dependiendo de la propia estructura dental (3-5).

Luego del blanqueamiento dental se generan leves alteraciones morfológicas en la superficie dental, debido a que los peróxidos crean microporosidades en ésta. Los cambios no son observables a simple vista, sin embargo, pueden predisponer a la pigmentación (6).
El objetivo de este artículo de revisión fue resaltar la evidencia científica sobre los mitos más comunes en el blanqueamiento dental y aclarar los puntos en los que el profesional odontólogo puede tener dudas. Para ello se discutirá la relación del blanqueamiento dental con la ingesta de bebidas, alimentos y hábitos que pueden pigmentar los dientes.

\section{"Bebidas oscuras manchan los dientes”}

Una de las principales indicaciones brindadas al paciente que recibe tratamiento de blanqueamiento dental es "No tomes bebidas que tengan colorantes". Algunas bebidas y colorantes artificiales de alimentos son responsables del oscurecimiento y pigmentación de los dientes, esto incluye bebidas como: el café, vino tinto, té, gaseosas, bebidas energizantes, entre otros (3).

Se ha encontrado información contradictoria en diversos estudios sobre el estado final de la superficie del esmalte dental luego del blanqueamiento, algunos autores refieren que no hay cambios significativos en la micro estructura superficial del esmalte luego del tratamiento de blanqueamiento (7). Sin embargo, otros demuestran cambios en la morfología de la superficie. Estos cambios van a depender de la sustancia utilizada, la concentración y el tiempo; la susceptibilidad a la pigmentación no sólo puede ser relacionada a la generación de superficies rugosas, existen otras consideraciones que debemos tener en cuenta como la composición del esmalte, la absorción de agua, alteraciones en la permeabilidad e irregularidades, todas estas pueden facilitar la acumulación de pigmentos $(6,8)$.

Una de las principales bebidas contraindicadas es el café. "No tomar café, el café mancha los dientes", es una recomendación común en el consultorio. Sin embargo, en la literatura se encuentra que si bien el café pigmenta los dientes, este no va a comprometer el resultado del blanqueamiento dental. Sobre esta recomendación Attia y col. evaluaron el cambio 
de color en dientes humanos y bovinos expuestos a soluciones de café durante el blanqueamiento; los especímenes fueron blanqueados con peróxido de carbamida al $16 \%$ en un periodo de seis horas diarias durante 28 días. La mitad de las muestras fueron separadas para sumergirlas en solución de café durante el blanqueamiento mientras que las otras muestras solo recibieron el tratamiento blanqueador. El color se evaluó mediante un análisis de fotoreflectancia durante el blanqueamiento, a los 7, 14 y 28 días y otra evaluación post-blanqueamiento a los 7, 15 y 30 días de terminado el tratamiento. Encontraron que el café no influyó en el resultado inmediato del blanqueamiento. Sin embargo, en la evaluación post-blanqueamiento se encontró que las muestras que recibieron café fueron más inestables en el color (8). Concluyeron que el consumo del café durante el blanqueamiento dental puede influir en la estabilidad del color después de este tratamiento.

Por otro lado, Liporoni y col,. quienes evaluaron la susceptibilidad a la pigmentación por café del esmalte blanqueado en diferentes periodos de tiempo inmediatamente después del blanqueamiento (1). Se expusieron muestras de esmalte bovino a peróxido de hidrógeno al 35\% y luego del tratamiento colocaron las muestras en café, un grupo se mantuvo en el café durante 30 min y el otro durante $150 \mathrm{~min}$, el color fue evaluado mediante espectroscopia de fotoreflectancia, no encontraron variaciones en el color después de sumergir las muestras en café, por lo que los autores concluyen que el café no afecta el resultado del blanqueamiento dental (1).

Bazzi y col., evaluaron el potencial del peróxido de hidrógeno al 6\% para eliminar pigmentaciones de café en esmalte bovino; sumergieron los especímenes en café por 72 hrs, y luego utilizaron peróxido de hidrógeno al 6\% una hora por día durante $21 \mathrm{~d}$, para finalmente volver a sumergirlos en café por 72 hrs, se realizaron mediciones de color después de cada procedimiento, las muestras presentaron variaciones significativas en los cambios de color después del blanqueamiento, sin embargo, la coloración aumentó después de la repigmentación (9). Los autores concluyen que el blanqueamiento es efectivo para eliminar las manchas causadas por café, pero si el consumo de éste es continuo después del blanqueamiento, el diente puede volver a pigmentarse (9).

En concordancia con este estudio encontramos a
Côrtes y col., quienes evaluaron la influencia del café durante y después del blanqueamiento dental con peróxido de carbamida al 10, 15 y 20\% en especímenes de esmalte dental humano, para ello realizaron una aplicación diaria de 4 horas del agente por 22 días, para luego exponerlos diariamente a café por $15 \mathrm{mi}-$ nutos; la estabilidad del color fue medida mediante un espectrofotómetro en los días 1, 7, 15, 22, 29 y 52 desde la primera aplicación; encontraron que durante el blanqueamiento la exposición a café no tuvo diferencia significativa en relación a los valores de reflectancia, es decir el blanqueamiento fue efectivo incluso durante la exposición a café; luego de los 22 días de tratamiento se encontró una disminución de la reflectancia, es decir el consumo de café luego del blanqueamiento modificó estos valores (10). Por lo que concluyeron que los pacientes pueden tomar café durante el tratamiento de blanqueamiento sin afectar los resultados, sin embargo, si continúan con esos hábitos presentarán variaciones en la estabilidad de color (10).

Estos resultados también fueron encontrados en un estudio in vivo realizado por Rezende y col., quienes evaluaron el efecto del café durante el blanqueamiento dental ambulatorio, los participantes fueron divididos en dos grupos; ambos fueron tratados con peróxido de carbamida al 16\% durante tres semanas; durante el tratamiento a un grupo se le indicó hacer enjuagues con café por $30 \mathrm{~s}$ cuatro veces al día y al segundo grupo se le indicó no beber café durante el tratamiento, el color de los dientes fue evaluado mediante el espectofotómetroEasyshade (VITA, Alemania) y visualmente con la escala Vita ordenada por valor; no encontraron diferencias estadísticamente significativas entre los dos grupos, concluyendo que el consumo de café durante el blanqueamiento no influye en el resultado final del tratamiento (11).

En base a la evidencia citada se puede concluir que el consumo frecuente de café sí pigmenta los dientes. Los peróxidos son efectivos para contrarrestar esta pigmentación independientemente del agente blanqueador utilizado y que el consumo de café durante el blanqueamiento no influye en el resultado final inmediato, sin embargo, si el paciente continúa consumiendo esta bebida después del blanqueamiento puede existir una repigmentación.

Otra bebida que se contraindica durante el blan- 
queamiento dental es el vino tinto. Berger y col. observaron que el blanqueamiento dental con peróxido de hidrógeno al 35\% aumenta la susceptibilidad de pigmentación del esmalte cuando es expuesto a vino tinto, el peróxido de hidrógeno pudo haber promovido alteraciones en la superficie del esmalte, lo cual aumentó la susceptibilidad a la pigmentación por vino tinto (12). En tal sentido los autores no recomiendan que los pacientes beban vino tinto durante el tratamiento de blanqueamiento porque durante este periodo el esmalte se encuentra más propenso a pigmentaciones por esta bebida (1).

Otro dato importante fue presentado por Côrtes y col., quienes evaluaron la influencia del vino tinto durante y después del blanqueamiento dental con peróxido de carbamida al 10, 15 y $20 \%$; ellos encontraron que no hubo diferencia significativa en valores de reflectancia en especímenes de esmalte expuestos a vino tinto durante el blanqueamiento dental; sin embargo, luego de finalizar el tratamiento se encontró una disminución importante de los valores de reflectancia, siendo la diferencia mayor que los encontrados con café. Concluyeron que los pacientes deben evitar el consumo de vino tinto después del tratamiento de blanqueamiento dental (10).

De Araújo y col. evaluaron la estabilidad de color en el esmalte dental sometido a blanqueamiento con peróxido de carbamida al 10\% combinado con soluciones pigmentantes (gaseosa, chocolate derretido y vino tinto), encontrando que el esmalte expuesto al vino tinto mostró la menor estabilidad de color (13).

En base a la evidencia científica citada se puede concluir que el consumo frecuente de vino tinto sí pigmenta los dientes, incluso la variación de color va a ser mayor que la causada por café. La mayor pigmentación, o menor estabilidad de color, cuando hay exposición a vino tinto se debe al $\mathrm{pH}$ ácido de la sustancia, por su contenido de etanol y a los pigmentos presentes en su composición $(10,12,14)$. Por lo tanto, se debe contraindicar el consumo de vino tinto durante el tratamiento de blanqueamiento dental éste último es efectivo para remover las pigmentaciones, causadas por el vino tinto. Sin embargo, si el paciente continúa con este hábito posterior al blanqueamiento el color puede llegar a ser más oscuro que el color inicial. El té también es considerado una bebida responsable de pigmentación de los dientes (15-17).
Karadas y col. compararon la pigmentación en esmalte dental luego de blanqueamiento con peróxido de carbamida al 10\% durante 6 hrs al día por 14 d, expusieron 4 sustancias diferentes (Café, Té, Coca-Cola y Vino tinto) por 15 min, 6 hrs, 1 semana y 1 mes.

Concluyeron que los pacientes deberían evitar el consumo de vino, té y Coca-Cola luego del tratamiento de blanqueamiento dental (3).

Por otro lado Azer y col., evaluaron el efecto del pigmento rojo 40, que se encuentra en diversos alimentos como cereales (Trix y FruitLoops) y bebidas (Gatorade), sobre la efectividad del blanqueamiento dental. Expusieron esmalte dental a peróxido de carbamida al 20\% durante por $10 \mathrm{hrs,} \mathrm{luego} \mathrm{estos} \mathrm{fueron}$ inmersos en $0,025 \%$ de colorante rojo 40 por 4 hrs, luego fueron pulidos con piedra pómez. Obtuvieron dos mediciones con un espectoradiómetro para evaluar diferencias en CIE a* (parámetro de rojos). Los valores obtenidos luego del pulido se consideraron como pigmentaciones intrínsecas y la diferencia entre los valores posteriores al pulido y valores antes del pulido se consideraron como pigmentaciones extrínsecas. Luego de la aplicación del pigmento rojo 40 después del blanqueamiento se obtuvo que sólo los valores de pigmentaciones extrínsecas aumentaron, sin embargo no se encontró diferencia estadísticamente significativa en cuanto a pigmentaciones intrínsecas (2). Esto es importante ya que significa que pueden ser removidas con un pulido. Los autores concluyen que es necesaria mayor investigación, al mismo tiempo sugirieron que para optimizar los efectos del blanqueamiento sería beneficioso la reducción de alimentos con colorantes.

\section{"No fumes durante el blanqueamiento"}

Otra de las indicaciones es la de no fumar durante el tratamiento de blanqueamiento dental; la principal preocupación se encuentra en los radicales libres que el cigarro genera, ya que éstos son considerados dañinos y cancerígenos, se cree que el peróxido de hidrógeno daña al paciente. Hannig y col. midieron la cantidad residual de radicales de peróxido de hidrógeno en pacientes fumadores y no fumadores. Realizaron un estudio in vivo, en el que utilizaron un sistema de análisis fotométrico basado en una reacción química para evaluar la cantidad de residuos de peróxido de hidrógeno durante el blanqueamiento dental. Encontraron que ser fumador no impactó en la capacidad de 
la boca de degradar los peróxidos, no encontraron diferencias entre la cantidad de peróxido en saliva entre pacientes fumadores y no fumadores (18). Además las concentraciones no pasaron del $20 \%$ de la dosis diaria segura (0.26 mg kg-1 día-1). Se puede concluir que el blanqueamiento dental en combinación con fumar es teóricamente seguro. Sin embargo, no debemos propiciar este hábito.

La otra preocupación con el hábito de fumar es la capacidad que podría tener el humo del cigarro en manchar los tejidos dentales. El humo de cigarro está compuesto de aire, agua, monóxido de carbono y dióxido de carbono. Sin embargo, a medida que el cigarro arde, los componentes como el alquitrán y azúcares se adhieren al humo del cigarro (19). Algunos autores refieren que la inclusión de estos pigmentos en el humo del cigarro seria la causa de las pigmentaciones debido a su color y a su habilidad para adherirse a la superficie del diente. Bazzi y col. evaluaron la habilidad del blanqueamiento dental para remover manchas de humo del cigarrillo en esmalte bovino. Estas se expusieron al humo de cigarrillo en una máquina de fumar. Se dividió cada grupo en dos subgrupos y se sometieron las muestras a blanqueamiento ambulatorio o al cepillado de dientes simulado, seguido de otra medición de color. Los autores repitieron ambos procedimientos de pigmentación y realizaron una tercera medición de color. El blanqueamiento y el cepillado mostraron una reducción significativa en el cambio de color, por lo que se debe recomendar el cepillado y el blanqueamiento dental en pacientes fumadores (8). Se concluye que el humo del cigarro sí mancha los dientes, sin embargo este tipo de manchas parece ser superficial. El blanqueamiento dental y el cepillado dental es efectivo para remover manchas extrínsecas causadas por este.

\section{“El blanqueamiento debilita los dientes?”}

Azer y col. realizaron un estudio in vitro con molares humanos. Se realizaron nanoindentaciones para evaluar la nanodureza y el módulo de elasticidad luego de la aplicación de peróxido de carbamida al 22\%, peróxido de hidrógeno al 9\% o productos over-thecounter (tiras con peróxido de hidrógeno al 10\% y $14 \%)$. Ellos encontraron que en ambos casos la microdureza y el módulo elástico decaía luego de la acción del agente blanqueador, la disminución fue mayor cuando la concentración de agente blanqueador fue más elevada (2), de lo que se deduce que a mayor concentración de peróxido de hidrógeno disminuye la dureza del esmalte.

Sin embargo, Ren y col. estudiaron el efecto del peróxido de hidrógeno al 6\% sobre la superficie de esmalte dental humano comparándolo con el efecto de la exposición al jugo de naranja. Para ello, ambos grupos fueron expuestos durante 20 min por 5 días a cada sustancia. Evaluaron la microdureza y la topografía superficial, encontrando que la microdureza del esmalte decreció en un 84\% luego de la inmersión al jugo de naranja y sólo se encontraron cambios en la topografía en este mismo grupo. No hubo diferencias significativas con respecto a microdureza ni cambios en la topografía en los grupos que recibieron blanqueamiento, ni en el grupo control (20). De este se puede deducir que si se selecciona adecuadamente el agente blanqueador el tratamiento va a ser seguro sin causar una modificación de la estructura del esmalte dental, comparable con la acción erosiva del juego de naranja.

De Araújo y col. evaluaron la pérdida mineral en esmalte dental sometido a blanqueamiento dental con peróxido de carbamida al $10 \%$ combinado con una solución pigmentante. Las soluciones fueron gaseosa, chocolate derretido y vino tinto. El grupo de vino tinto y gaseosa presentaron mayor pérdida de mineral comparado con los otros grupos (13). Los autores relacionan el cambio en la pérdida de mineral con la modificación de la superficie y la susceptibilidad a la pigmentación.

Sin embargo, algunos autores han reportado que no hay significancia en la pérdida de calcio o fostato, ni en la reducción de la microdureza en dientes blanqueados ya que la saliva tiene un rol importante en el proceso de intercambio mineral del esmalte.

\section{CONCLUSIONES}

Consumir café o té no afecta el resultado inmediato del blanqueamiento dental, pero si influye en la estabilidad de los resultados.

Consumir vino tinto durante el blanqueamiento influye negativamente en el resultado.

El tratamiento de blanqueamiento dental es efectivo para remover manchas producidas por el humo de cigarro. 
El blanqueamiento no causa modificaciones permanentes en la estructura del esmalte dental.

\section{Correspondencia}

Lidia Yileng Tay Chu Jon

Correo electrónico: lidia.tay.c@upch.pe

\section{REFERENCIAS BIBLIOGRÁFICAS}

1. Liporoni PC, Souto CM, Pazinatto RB, et al. Enamel susceptibility to coffee and red wine staining at different intervals elapsed from bleaching: a photoreflectance spectrophotometry analysis. Photomed Laser Surg. 2010;28(2):105-9.

2. Azer SS, Hague AL, Johnston WM. Effect of bleaching on tooth discolouration from food colourant in vitro. J Dent. 2011;39(3):52-6.

3. Karadas M, Seven N. The effect of different drinks on tooth color after home bleaching. Eur J Dent. 2014;8(2):249-53.

4. Erdemir U, Yildiz E, Eren MM, Ozel S. Surface hardness of different restorative materials after long-term immersion in sports and energy drinks. Dent Mater J. 2012;31(5):729-36.

5. Azer SS, Machado C, Sanchez E, Rashid R. Effect of home bleaching systems on enamel nanohardness and elastic modulus. J Dent. 2009;37(3):185-90.

6. Dahl JE, Pallesen U. Tooth bleaching - A critical review of the biological aspects. Crit Rev Oral Biol Med. 2003;14(4):292-304.

7. Maia E, Baratieri LN, Caldeira de Andrada MA, Monteiro S Jr, Vieira LC. The influence of two home -applied bleaching agents on enamel microhardness: an in situ study. J Dent. 2008;36(1):2-7.

8. Carey CM. Tooth whitening: what we now know. J Evid Based Dent Pract. 2014;14(1):70-6.

9. Bazzi JZ, Bindo MJ, Rached RN, Mazur RF, Vieira $S$, de Souza EM. The effect of at-home bleaching and toothbrushing on removal of coffee and cigarette smoke stains and color stability of enamel. J Am DentAssoc. 2012;143(5):1-7.

10. Côrtes G, Pini NP, Lima DA, et al. Influence of coffee and red wine on tooth color during and after bleaching. Acta OdontolScand. 2013;71(6):1475-80.

11. Rezende M, Loguercio AD, Reis A, Kossatz S. Clinical effects of exposure to coffee during at-home vital bleaching. Oper Dent. 2013;38(6):229-36.

12. Berger SB, Coelho AS, Oliveira VA, Cavalli V, Giannini M. Enamel susceptibility to red wine staining after 35\% hydrogen peroxide bleaching. J Appl Oral Sci. 2008;16(3):201-4.

13. De Araujo LS, Dos Santos PH, Anchieta RB, et al. Mineral loss and color change of enamel after blea- ching and staining solutions combination.J Biomed Opt. 2013; 18(10):108004.

14. Attia ML, Aguiar FH, Mathias P, Ambrosano GM, Fontes CM, Liporoni PC. The effect of coffee solution on tooth color during home bleaching applications. Am J Dent. 2009;22(3):175-9.

15. Griffiths CE, Bailey JR, Jarad FD, Youngson CC. An investigation into most effective method of treating stained teeth: an in vitro study. J Dent. 2008;36(1):54-62.

16. Adeyemi AA, Jarad FD, de Josselin de Jong E, Pender $\mathrm{N}$, Higham SM. The evaluation of a novel method comparing quantitative light-induced fluorescence (QLF) with spectrophotometry to assess staining and bleaching of teeth. Clin Oral Investig. 2010;14(1):19-25.

17. D’Arce MB, Lima DA, Aguiar FH, Bertoldo CE, Ambrosano GM, Lovadino JR. Effectiveness of dental bleaching in depth after using different bleaching agents. J Clin Exp Dent. 2013;5(2):100-7.

18. Hannig C, Willenbücher S, Becker K, Mahony C, Attin T. Recovery of peroxides in saliva during home bleaching--influence of smoking. J Oral Rehabil. 2006;33(7):533-41.

19. Andrade L, Rodrigues T, Ramos P, Nóbrega A, Mathias P. Changes in Water Sorption and Solubility of Dental Adhesive Systems after Cigarette Smoke. ISRN Dent. 2013; 2013(1):1-5

20. Ren YF, Amin A, Malmstrom H. Effects of tooth whitening and orange juice on surface properties of dental enamel. J Dent. 2009;37(6):424-31.

Recibido : 03/11/2014

Aceptado: 15/02/2015 